\title{
The effect of milling duration on silicon dioxide characterization
}

\author{
Mohd Aizat Hafiz Mohd Sohor ${ }^{1 *}$, Mazli Mustapha ${ }^{1}$, and Othman Mamat ${ }^{1}$ \\ ${ }^{1}$ Mechanical Engineering Department, Universiti Teknologi PETRONAS (UTP), 32610 Seri \\ Iskandar, Perak, Malaysia
}

\begin{abstract}
This paper reports the study of milling duration on silicon dioxide characterization. The silicon dioxide has undergone mechanical milling treatment from 0 to 100 hours. The product after milling process was characterized by using scanning electron microscopy and X-ray diffraction. Williamson-Hall plot technique was used in this study to analyse the silicon dioxide crystallite size and lattice strain. The longer milling duration resulted in the reduction of crystallite size and increase in lattice strain.
\end{abstract}

\section{Introduction}

Silicon carbide $(\mathrm{SiC})$ is an interesting ceramic material that has unique properties in mechanical, optical and electrical aspects. These special properties and characteristics have made $\mathrm{SiC}$ to be widely applied in industrial and engineering fields. Carbothermal reduction process involved long hours of heating raw materials at extremely high temperatures which was around $2200-3300^{\circ} \mathrm{C}$. Quartz sand and petroleum coke were used as precursor materials for this technique [1]. Large particles powder was produced by this process because of high temperature and long heating hours. Therefore, extensive mechanical milling (MM) of raw materials was needed to produce smaller diameter size and will aid the reaction to reduce sintering temperature [2]. This paper aims to present the effect of milling duration on silicon dioxide characterization.

\section{Experimental procedure}

The precursor materials of silicon and carbon were prepared from amorphous silicon dioxide, $\mathrm{SiO}_{2}$ (Johore silica, 99.5\% purity) and carbon black powder from petroleum coke was originally from Petronas refinery. Silicon dioxide was performed mechanical treatment in a stainless-steel jar by using iron as ball milling media. The ball to powder ratio was set to 20:1 with $200 \mathrm{rpm}$ grinding speed. The mechanically milled silica and carbon were mixed with $\approx 10 \%$ silica and $\approx 60 \%$ carbon beyond the stoichiometric ratio $\mathrm{C} / \mathrm{SiO}_{2}$ of $3: 1$ and were placed in the rotary mixture.

\footnotetext{
*Corresponding author: mohd.aizat_g03388@utp.edu.my
} 
The morphology of samples was analyzed under scanning electron microscopy (SEM) (VPFESEM, Zeiss Supra55 VP) and chemical composition was analysed by energy dispersive spectroscopy (EDS). The phase composition was determined by Bruker model D8 Advanced X-ray Diffractometer $\left(\mathrm{Cu} \mathrm{K}_{\alpha}\right.$ radiation, $\left.\lambda=0.15406 \mathrm{~nm}\right)$ from Braggs angle $(2 \theta)$ of 10 to $90^{\circ}$ with steps of $0.040^{\circ}$. Phase identification of the powders were carried out using EVA software. Positions of the peaks in a diffraction profile are integral in X-ray diffraction (XRD) when conducting phase identification. The relative intensities of a peak in a diffraction profile as well as relative intensities of these peaks also influence it. Additional vital information can be gleaned from the shapes of the peaks. In particular, the width of a peak indicates the amplitude of thermal oscillations of the atoms at their regular lattice sites. Furthermore, it is an indication of vacancy and element concentrations impurities and even plastic deformation; any factor which affects the distribution of d-spacings.

The broadening of a peak represents the crystallite size. Scherrer equation satisfies the Bragg condition for non-adjacent diffraction planes as it explains peak broadening in terms of incident beam divergence [3]. The peak width (described as full width at half maximum peak intensity (FWHM)), peak position and wavelength are used in a function to calculate crystallite size once instrument effects have been excluded.

The Williamson Hall plot method's is based on a Fourier deconvolution of the measured peaks and the instrument broadening to obtain the true diffraction profile [3]-[6]. This method can yield both the crystallite size distribution and lattice macrostrain. Uniform residual strain will lead to a parallel shift in the x-ray diffraction peaks for the specimens that experienced large plastic deformation. Conversely, the broadening of x-ray diffraction peaks are caused by varying residual strain. Since the broadening parameter of the x-ray peaks is due to strain effect as well as size effect, it could then be written as following:

$$
B_{s}=\frac{0.9 \lambda}{d \cos \theta}+\eta \tan \theta
$$

By multiplying $\cos \theta$ to (1), the formula becomes:

$$
B_{s} \cos \theta=\frac{k \lambda}{d}+\eta \sin \theta
$$

where $B_{s}$ refers to full width at half maximum (FWHM), $\theta$ the Bragg angle, $d$ is the crystallite size, $\eta$ the lattice strain, $\lambda$ the x-ray wavelength, $k$ is Scherrer constant $(=0.9)$. The instrumental broadening $\left(B_{i}\right)$ should be corrected by the following equation:

$$
B_{s}^{2}=B_{e}^{2}-B_{2}^{2}
$$

where $B_{e}$ is the FWHM of the measured XRD peak. Thus, when $\mathrm{B} \cos \theta$ is plotted against $\sin \theta$, a straight line will be obtained with the slope as $\eta$ (lattice strain) and the intercept being $k \lambda d$ where crystallite size, $d$, can be calculated by using this method. 


\section{Results and discussions}

\subsection{Influence of mechanical milling treatment on silicon dioxide}

The XRD patterns shown in Figure 1 had different duration of grinding which ranged from 0 hour to 100 hours while Fig. 2 (a) - (f) shows the surface morphology of the silica sand which was subjected to various milling time. For reference, the highest peak for Un-MM silica sand is used and it is at $26.2^{\circ}$ ( $2 \theta$ angle). After 20 hours of grinding, it has shown that the intensity of the peak had reduced up to $89.5 \%$ whereas $98.3 \%$ was achieved after 100 hours of grinding. The increment of broadening effect at the peak base of the spectrum is caused by longer mechanical milling time in order to reduce particle size (MM) silica sand. Similar finding by Suryanarayana where talc sample is used instead has shown the same XRD pattern [3]. Szewezak et al have reported that the maximum size reduction limit for a particle is equivalent to the size of tough-brittle transition which lead to plastic deformation rather than breakage of particle and it is accompanied by the growth of structure distortions [6].

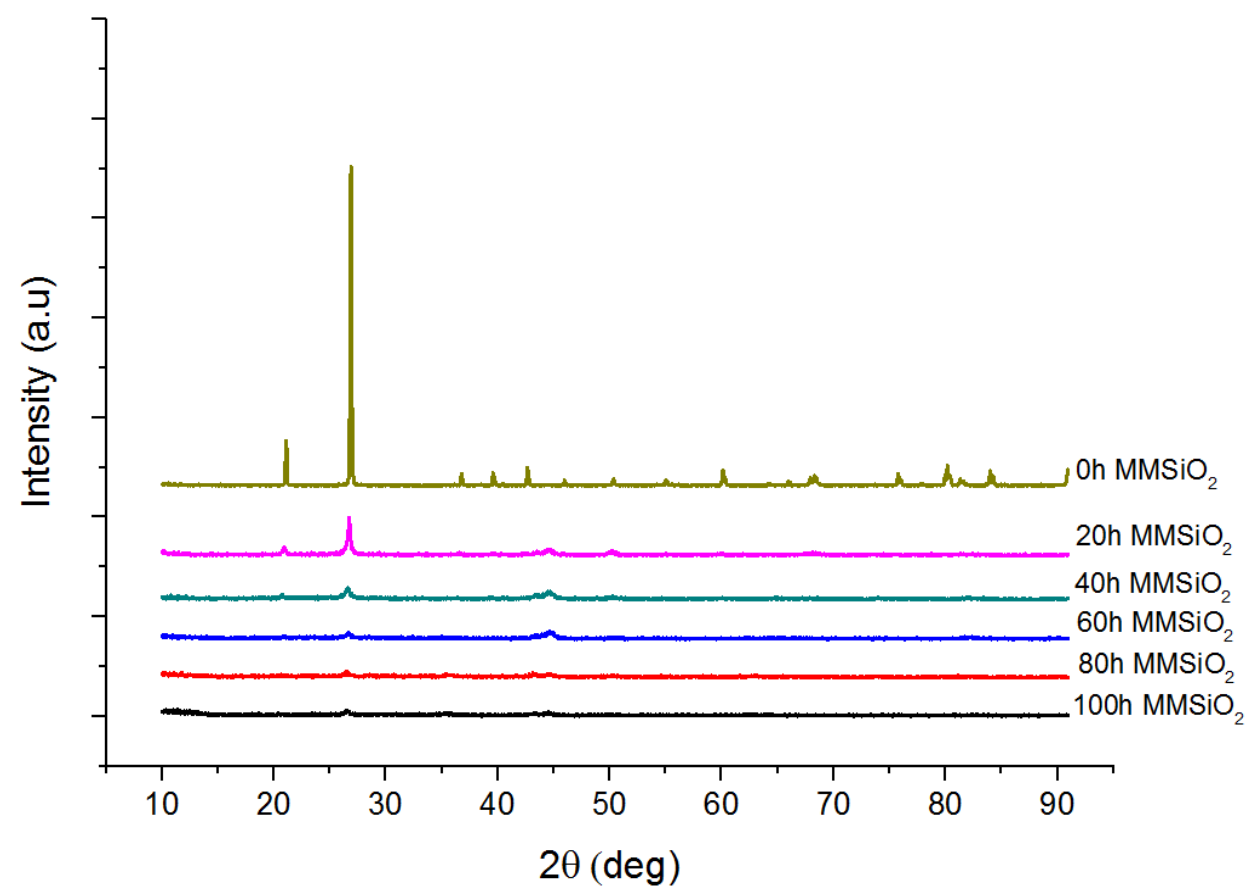

Fig. 1. X-ray diffraction pattern of $\mathrm{SiO}_{2}$ at various milling duration 


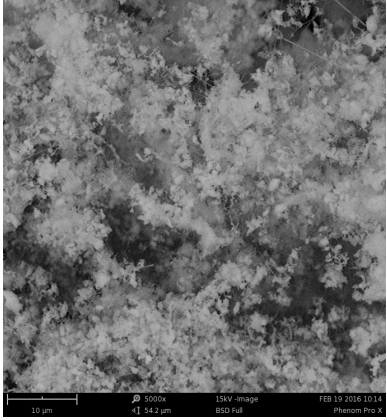

(a)

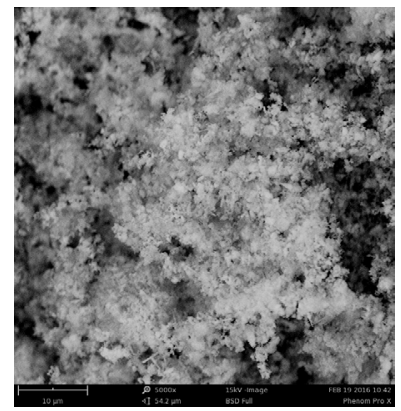

(d)

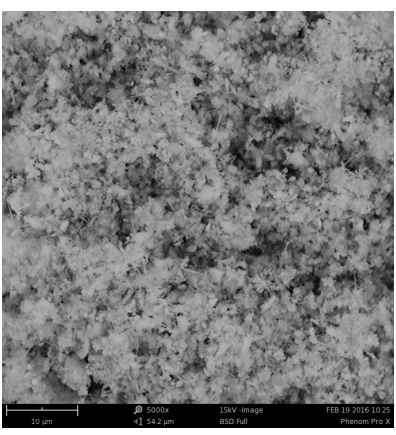

(b)

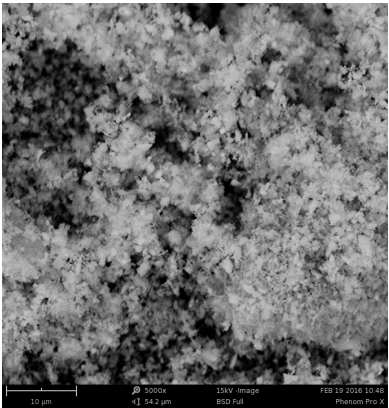

(e)

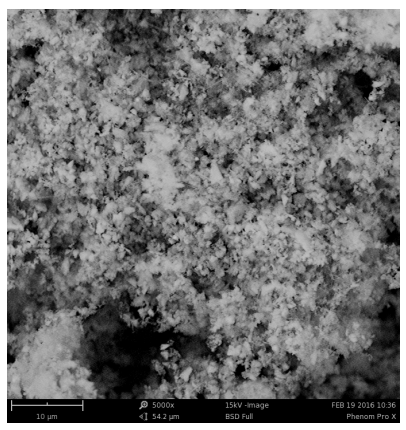

(c)

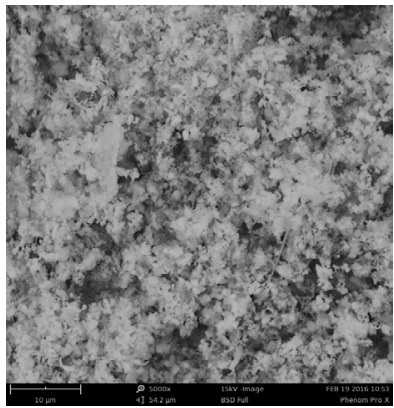

(f)

Fig. 2. The SEM images of $\mathrm{SiO}_{2}$ sand after undergoing mechanical treatment at various milling time: (a) $0 \mathrm{~h} \mathrm{(b)} 20 \mathrm{~h} \mathrm{(c)} 40 \mathrm{~h}$ (d) $60 \mathrm{~h} \mathrm{(e)} 80 \mathrm{~h}$ (f) $100 \mathrm{~h}$

Table 1 shows the intensity value at milling time ranging from 0 hour to 100 hours. It can be seen that the degree of random disorder in (MM) silica sand increases as milling time increases. At 100 hours of milling time, the degree of random disorder is about $98.3 \%$. This indicates that the mechanical milling process which is based on impact and friction among particles not only causes size reduction but also produces important changes in the physio chemical properties of solid. This leads to structural alteration because of loss of regularity in the crystalline network. The observation of line broadening in the X-ray diffraction spectra is an important method that was used for distortion investigation. The degree of random disorder and lattice distortion of the particles are indicated by the intensity and half width of $\mathrm{X}$-ray lines in different patterns.

Table 1. Intensity value at different milling time

\begin{tabular}{|c|c|c|}
\hline Milling Time (h) & Intensity (a.u) & Random Disorder (\%) \\
\hline 0 & 1818 & - \\
\hline 20 & 191 & 89.5 \\
\hline 40 & 60 & 96.7 \\
\hline 60 & 39 & 97.9 \\
\hline 80 & 33 & 98.2 \\
\hline 100 & 30 & 98.3 \\
\hline
\end{tabular}




\subsection{Crystallite size and microstrain}

The crystallite size, $D$ and the microstrain, $\eta$ for the fabricated foam at various $\mathrm{SiO}_{2}$ after milling process were calculated from the Williamson-Hall plot (Fig. 3) and tabulated in Table 2. The table reveals that as milling time increases, the crystallite size decreases. Abdoli et al. also reported a similar finding whereby a ductile $\mathrm{Al}$ has a higher chance to plastically deform which can lead to grain refinement and changes in lattice distortion [7]. They further added that low stiffness or high work hardening capacity powders will densify more effectively. The increment of milling time leads to the increase of microstrain which can be attributed to $\mathrm{SiO}_{2}$ milling. During the process, they were in a complicated stress strain state after pressure was being applied by the iron ball which acted as milling media inside the stainless-steel jar.

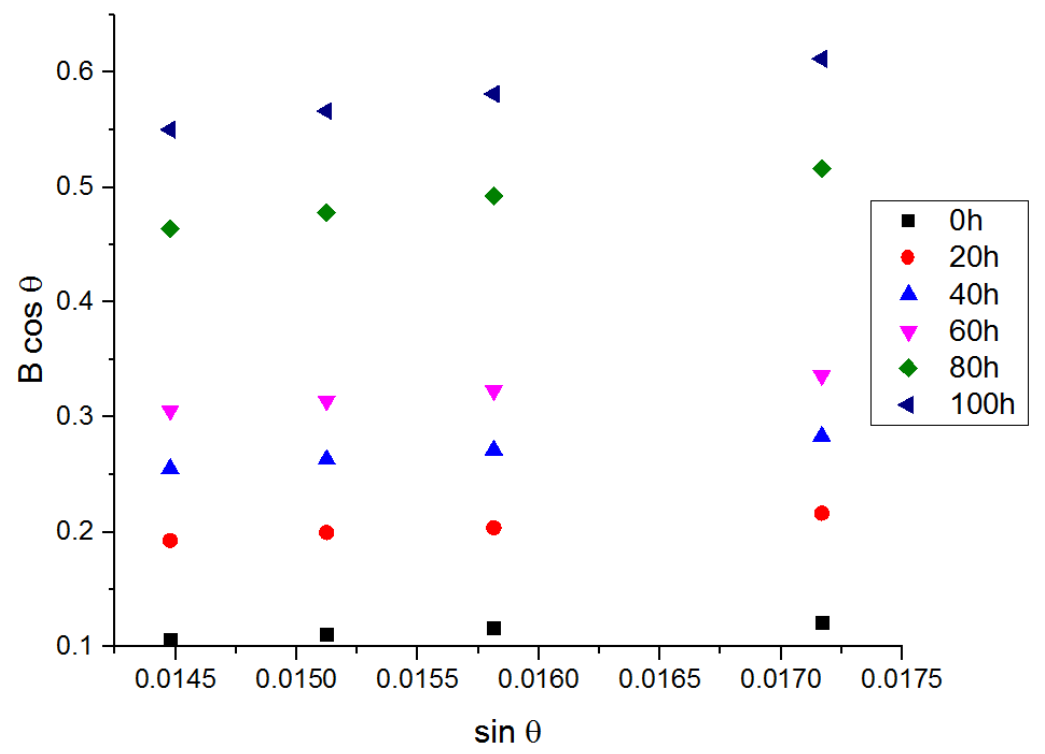

Fig. 3. Williamson-Hall plot for $\mathrm{SiO}_{2}$ with different milling duration

Table 2. Lattice strain and crystallite size mechanically milled $\mathrm{SiO}_{2}$ calculated from Williamson-Hall plot method

\begin{tabular}{|c|c|c|}
\hline MMSiO $_{2}(\mathbf{h})$ & $\begin{array}{c}\text { MMSiO}_{2} \text {-Crystalline } \\
\text { Size }(\boldsymbol{\mu m})\end{array}$ & Lattice Strain (\%) \\
\hline 0 & 4.95 & 5.47 \\
\hline 20 & 2.04 & 8.62 \\
\hline 40 & 1.31 & 10.4 \\
\hline 60 & 0.96 & 11.23 \\
\hline 80 & 0.76 & 19.84 \\
\hline 100 & 0.63 & 22.74 \\
\hline
\end{tabular}




\section{Conclusion}

In summary, the preparation of precursor material namely $\mathrm{SiO}_{2}$ has been done successfully through mechanically milled treatment at various times. XRD pattern showed that the intensity peak lowered with longer duration of $\mathrm{SiO}_{2}$ milling time. Similarly, the increase of mechanical milling time caused the size reduction of $\mathrm{SiO}_{2}$ crystalline and increase in $\mathrm{SiO}_{2}$ lattice strain.

The authors want to thank Universiti Teknologi PETRONAS for necessary support throughout the project. This project is financially funded by the Malaysian Ministry of Higher Education (MOHE) under the Fundamental Research Grant Scheme (FRGS) no. 0153AB-K82.

\section{References}

1. S. Cetinkaya and S. Eroglu, J. Eur. Ceram. Soc., 31, no. 5, pp. 869-876, (2011)

2. B. M. Moshtaghioun, A. Monshi, M. H. Abbasi, and F. Karimzadeh, Int. J. Refract. Met. Hard Mater., 29, no. 6, pp. 645-650, (2011)

3. C. Suryanarayana, Progress in Materials Science, 46, no. 1-2. pp. 1-184, (2001)

4. J. S. Lee, Y. K. Byeun, S. H. Lee, and S. C. Choi, J. Alloys Compd., 456, no. 1-2, pp. 257-263, (2008)

5. F. Charlot, E. Gaffet, B. Zeghmati, F. Bernard, and J. C. Niepce, Mater. Sci. Eng. A, 262, no. 1-2, pp. 279-288, (1999)

6. E. Szewczak and J. W. Wyrzykowski, Nanostructured Mater.,12, no. 1, pp. 171-174, (1999)

7. H. Abdoli, H. Farnoush, E. Salahi, and K. Pourazrang, Mater. Sci. Eng. A, 486, no. 12, pp. 580-584 (2008) 